Research Paper

\title{
The Structural Function of Nestin in Cell Body Softening is Correlated with Cancer Cell Metastasis
}

\author{
Ayana Yamagishi ${ }^{1}$, Moe Susaki², Yuta Takano², Mei Mizusawa ${ }^{2}$, Mari Mishima $^{2}$, Masumi Iijima ${ }^{3,4}$, \\ Shun'ichi Kuroda4, Tomoko Okada1, Chikashi Nakamura1,2, \\ 1. Biomedical Research Institute, National Institute of Advanced Industrial Science and Technology (AIST), Central 5 1-1-1 Higashi, Tsukuba, Ibaraki, \\ 305-8565, Japan \\ 2. Department of Biotechnology and Life Science, Tokyo University of Agriculture and Technology, 2-24-16 Naka-cho, Koganei, Tokyo, 184-8588, Japan \\ 3. Department of Nutritional Science and Food Safety, Faculty of Applied Bioscience, Tokyo University of Agriculture, 1-1-1 Sakuragaoka, Setagaya-ku, \\ Tokyo, 156-8502, Japan \\ 4. Department of Biomolecular Science and Reaction, The Institute of Scientific and Industrial Research, Osaka University, 8-1 Mihogaoka, Ibaraki, Osaka \\ 567-0047, Japan \\ $\bowtie$ Corresponding author: Chikashi Nakamura. Tel.: +81-29-861-2445; fax: +81-29-861-3048; E-mail address: chikashi-nakamura@aist.go.jp
}

(c) Ivyspring International Publisher. This is an open access article distributed under the terms of the Creative Commons Attribution (CC BY-NC) license (https://creativecommons.org/licenses/by-nc/4.0/). See http://ivyspring.com/terms for full terms and conditions.

Received: 2019.01.23; Accepted: 2019.05.02; Published: 2019.06.02

\begin{abstract}
Intermediate filaments play significant roles in governing cell stiffness and invasive ability. Nestin is a type VI intermediate filament protein that is highly expressed in several high-metastatic cancer cells. Although inhibition of nestin expression was shown to reduce the metastatic capacity of tumor cells, the relationship between this protein and the mechanism of cancer cell metastasis remains unclear. Here, we show that nestin softens the cell body of the highly metastatic mouse breast cancer cell line FPIOSC2, thereby enhancing the metastasis capacity. Proximity ligation assay demonstrated increased binding between actin and vimentin in nestin knockout cells. Because nestin copolymerizes with vimentin and nestin has an extremely long tail domain in its C-terminal region, we hypothesized that the tail domain functions as a steric inhibitor of the vimentin-actin interaction and suppresses association of vimentin filaments with the cortical actin cytoskeleton, leading to reduced cell stiffness. To demonstrate this function, we mechanically pulled vimentin filaments in living cells using a nanoneedle modified with vimentin-specific antibodies under manipulation by atomic force microscopy (AFM). The tensile test revealed that mobility of vimentin filaments was increased by nestin expression in FPIOSC2 cells.
\end{abstract}

Key words: Nestin, Intermediate filament, Cell stiffness, Cancer metastasis, Atomic force microscope

\section{Introduction}

Nestin is a type VI intermediate filaments (IF) protein that was originally utilized as a marker of neural stem cells. The structure of nestin is similar to that of a typical IF, consisting of an a-helical structure within a central rod domain flanked by N-terminal head and C-terminal tail domains. The head domain in particular is essential for assembly of IF filaments, which are comprised of approximately eight protofilaments assembled from antiparallel tetramers of IF proteins. Owing to its relatively short head domain (7 amino acids), nestin assembly is suggested to require the formation of a heterodimer with a partner IF protein that has a regular head domain such as vimentin [1]. Meanwhile, nestin has a remarkably large tail domain (approximately 1,550 amino acids) which associates with various proteins [2-5]. While this tail is thought to potentially contain sites for interaction with other cytoskeletal proteins [6], the function of this domain remains unclear.

Recently, increased nestin expression was detected in several cancer cell lines [7-9]. Moreover, the five-year survival rate of breast cancer patients with tumors exhibiting nestin expression is significantly lower than those with tumors that do not express nestin [10]. In vitro, knockdown of nestin expression in prostate and colorectal cancer cells 
resulted in suppression of cell migration and invasion $[11,12]$. These data indicate that nestin expression promotes cancer cell metastasis; however, the precise role of nestin in this process remains unknown.

Previous studies reported that cells from cancer patients are softer than benign cells [13, 14]. Since cancer cells spread from a primary tumor to nearby tissue and organs in the metastasis process, a soft body is beneficial to move through the connective tissue. Cell mechanical properties are largely dependent on the cytoskeletal structure, which is composed of actin filaments, microtubules, and IF. These cytoskeletal proteins and linker proteins interact with one another to form a three-dimensional structure. In particular, IF proteins surrounding the nucleus form web-like structures and contribute to the maintenance of cell shape and stiffness [15, 16]. Notably, knockdown of vimentin expression resulted in loss of directional migration and impaired cell stiffness in the human breast cancer cell line MDA-MB-231 [15]. Meanwhile, keratin-free murine keratinocytes were found to exhibit higher cell deformability and increased invasiveness, compared to wild type cells [16]. These studies suggest that IFs play a significant role in mediating cell stiffness and invasion.

To evaluate the relationship between nestin and cell stiffness in the mechanism of cancer cell metastasis, we generated a nestin knockout cell by CRISPR/Cas9 genome editing system in the mouse breast cancer cell FP10SC2 [17] which exhibits high levels of motility and metastasis [17]. Our findings indicate that nestin promotes cancer cell metastasis by reducing cell stiffness.

\section{Results}

\section{Nestin knockout reduces cancer metastasis}

We generated a nestin gene knockout strain using the CRISPR/Cas9 system from FP10SC2 cell. As shown in Fig. S1A, the nestin expression level in FP10SC2 was more than three-fold higher than that observed in its parental cells, 4T1E [18]. Immunostaining of obtained clones indicated that nestin expression disappeared in some knockout strains, particularly strain SNKG8, which exhibited complete abrogation of nestin expression (Fig. S1B). By sequencing analysis, the target alleles of the strain were confirmed to be successfully disrupted. The SNKG8 strain was therefore chosen for subsequent experiments.

To examine the effects of nestin knockout on the in vivo metastatic activity of cells, FP10SC2 or nestin knockout SNKG8 cells were intravenously injected into mice. While all of the mice injected with FP10SC2 cells were dead within 14 days $(n=11)$, the survival rate of those injected with SNKG8 $(n=12)$ was significantly prolonged $(\mathrm{p}<0.0001$; Gehan-Breslow-Wilcoxon test) (Fig. 1A). Therefore, FP10SC2 cells were more malignant than the SNKG8 strain, suggesting that metastatic ability of FP10SC2 cells was moderated by nestin knockout.

To reveal the factor affecting in vivo metastatic ability in nestin knockout cells, we evaluated the cell motility of FP10SC2 and SNKG8 cells. The motility of single SNKG8 cells, calculated by manually tracking the total moving distance, was the same as that exhibited by FP10SC2 cells (Fig. 1B). The result indicates that inhibition of metastasis was not due to an increase in cell motility. Next, we examined whether nestin knockout affected cell invasion via transwell migration assay analysis. Compared to the parental cells, SNKG8 cells exhibited decreased migration through the transwell membrane to the lower chamber (Fig. 1C), indicating the nestin knockout resulted in reduced invasion ability. Furthermore, wound-healing assay analysis demonstrated that SNKG8 cells exhibited slower healing rates than FP10SC2 cells (Fig. 1D). Since there were no differences in cell motility between FP10SC2 and SNKG8, other causes were thought to be involved in the reduced invasion and migration observed in SNKG8 cells.

\section{Cell stiffness increased in nestin knockout cancer cell}

Next, we assessed cellular stiffness of FP10SC2 and SNKG8 cells by cell indentation tests using atomic force microscopy (AFM) and cylindrical-shaped AFM cantilever (Fig. S2). Compared to FP10SC2 cells, SNKG8 cells were associated with a 1.5-fold increase in Young's modulus (Fig. 2A), indicating that the knockout cells were stiffer than the parental cells. We also verified the stiffness in the SNKG8 transfected with nestin expression plasmid vector. Nestin expression in each cell was confirmed by immunostaining after the measurement of the stiffness. To exclude cells which overexpressed nestin, threshold value was set at the average fluorescent intensity derived from nestin plus four standard deviations of positive control, FP10SC2 (Fig. S3) cells. As a result, the stiffness in nestin-rescued cell was restored to that in FP10SC2 (Fig. 2A). Because cellular stiffness in nestin knockout cell significantly decreased by exogenous expression of nestin, the increase of the stiffness in SNKG8 is considered to be due to the nestin disruption. These results therefore suggest that the reduction in metastatic ability observed in SNKG8 cells was due, at least in part, to increased cellular stiffness. We also 
performed nestin knockout in human glioma cell KG-1-C. As a result, nestin knockout cell of KG-1-C exhibited significantly higher stiffness than that of

A

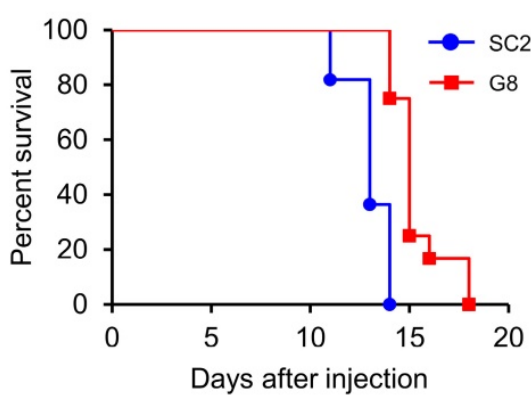

parental cell (Fig. 2A), indicating that increase of cellular stiffness by nestin knockout is not a cell-type-specific phenomenon.

B

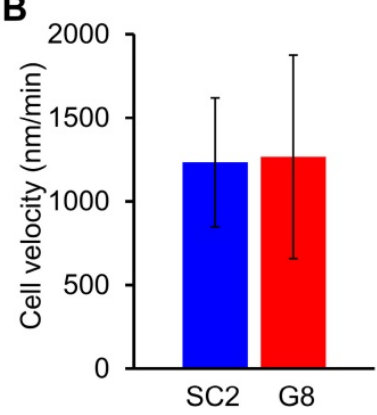

C

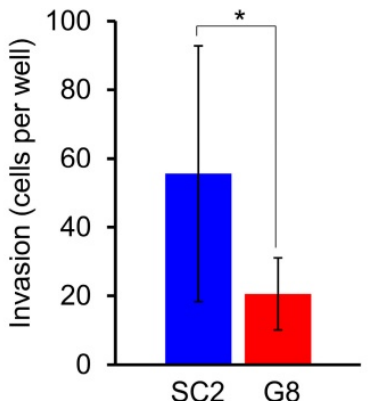

D

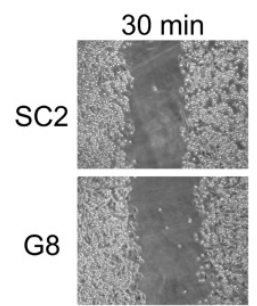

$6 \mathrm{~h}$
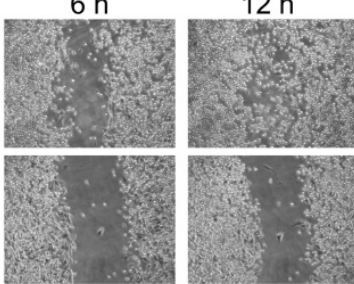

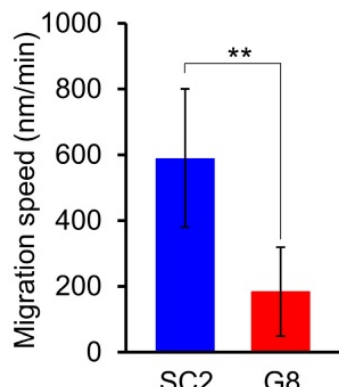

Figure 1. Analysis of the metastatic capacity of the nestin knockout strain. (A) Effects of subcutaneous injection of FP10SC2 (SC2, $n=11)$ or SNKG8 (G8, $n=12$ ) cells $\left(1 \times 10^{6}\right.$ cells/mouse) on the survival of female BALB/c mice. (B) Velocity of FP10SC2 $(n=21)$ and SNKG8 $(n=20)$ cell migration. Velocity was calculated by measuring the distance of movement of cells from the center of cell gravity over $30 \mathrm{~min}$. (C) Cell invasion assay analysis of FP10SC2 and SNKG8 cells. Cells that migrated through the Matrigel-coated transwell membrane to the lower chamber were enumerated $(n=7)$. (D) Representative images of the wound-healing capacity of FP10SC2 and SNKG8 cell monolayers $(n=3) ;{ }^{*} \mathrm{p}<0.05$, **p $<0.01$; Student's t-test.

A

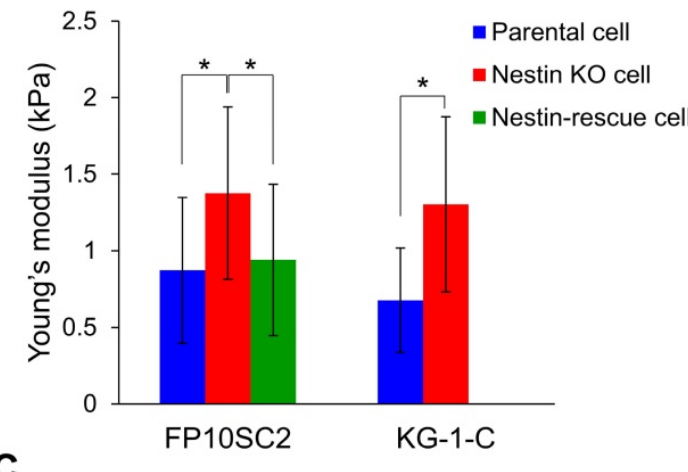

C

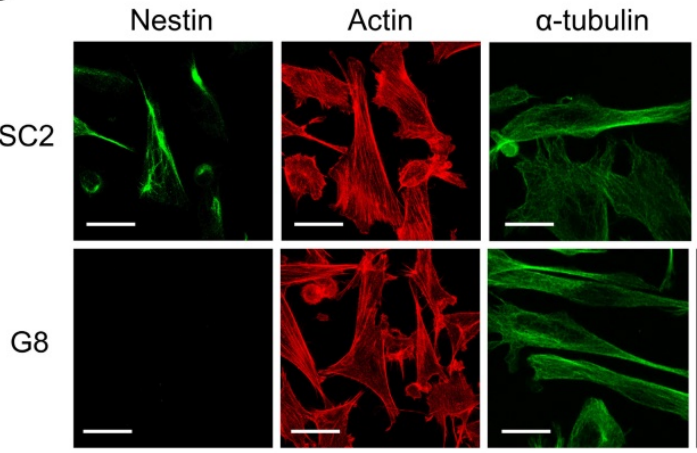

B
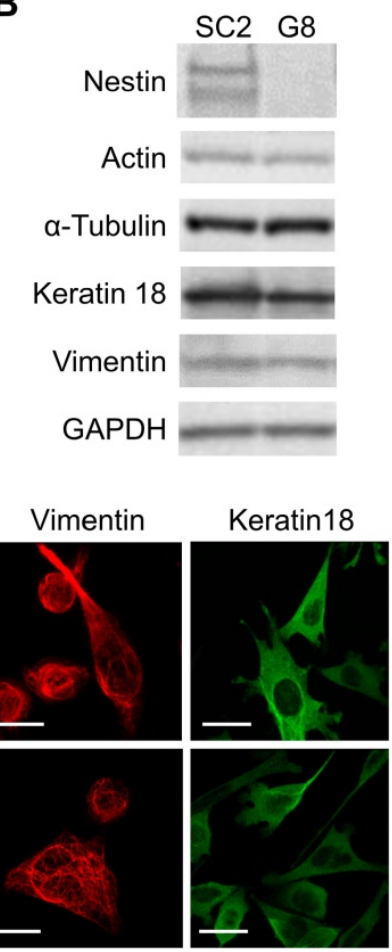

Figure 2. Effect of the nestin knockout on cell stiffness and cytoskeletal structure. (A) Evaluation of stiffness in nestin knockout cells using an atomic force microscope and a cylindrical-shaped cantilever; *p $<0.001$; Student's t-test. Nestin knockout cells of FP10SC2 $(n=45)$ or KG-1-C cells $(n=15)$ were seeded on the dish and measured by AFM. FPIOSC2 $(n=43)$ and KG-1-C $(n=21)$ cells were used as a control. For genetic rescue of nestin, SNKG8 cell was transfected with nestin expression vector and measured by AFM $(n=31)$. (B) Western blot analysis of actin, $\alpha$ tubulin, vimentin, keratin18, and nestin expression in parental FP10SC2 and SNKG8 cells. (C) Immunofluorescent images cytoskeletal protein staining in FP10SC2 and SNKG8 cells. Scale bar $=20 \mu \mathrm{m}$. 
The increase in cell stiffness caused by the nestin knockout could be due to changes in the levels of other cytoskeletal proteins and/or the cytoskeletal architecture. To investigate this concern, we evaluated actin, microtubule, vimentin, and keratin18 expression by immunofluorescence staining and western blot analyses. Notably, no significant differences in expression of these proteins were detected between FP10SC2 and SNKG8 cells by western blot (Fig. 2B), and immunostaining analysis indicated that the two cell lines exhibited similar cytoskeletal structures (Fig. 2C). Together, these findings suggest that the observed increase in cell stiffness was not due to increased expression of other cytoskeletal proteins, but due to the abrogation of nestin expression specifically, and that nestin therefore promotes cell body softening and likely influences the mechanical properties of the cytoskeleton.

We also investigated mRNA expression level in parental FP10SC2 and nestin knockout cell by RNA sequencing (RNA-seq) to assess impact of nestin knockout on other genes related to cancer metastasis. Based on a $\log 2$ fold change of RPKM (Reads Per Kilobase of exon per Million mapped reads) value, 20 most up-regulated and 20 most down-regulated genes by nestin knockout were listed in Table S1. Among these, the genes considered to be involved in the metastaticity are ADAMTS9 and DSG2 in the up-regulated genes, and ATAD2, RASEF, and Clic1 in the down-regulated genes. ADAMTS9 (a disintegrin-like and metalloprotease with thrombospondin type 1 motif 9) contributes to tumor suppression in gastric cancer cell [19]. Dsg2 (desmoglein-2), a desmosomal cadherin mediating cell-cell adhesion, is reported that an absent of Dsg2 associates with a progression of cancers [20]. In the down-regulated genes, ATAD2 (ATPase family AAA domain containing 2) and RASEF (Ras and EF-hand domain containing) have been reported to promote cancer cell proliferation [21, 22], and Clic1 (chloride intracellular channel protein 1) is considered to promote migration and invasion of cancer cell [23]. By western blotting, we investigated protein expression of Clic1 whose RPKM score is highest in the wild type, confirming that the Clic1 expression decreased actually in nestin knockout cells (Fig. S4A). Although the relationship between nestin and these genes is unclear now, it will be elucidated in further study.

It has been recently reported that nestin acts as an inhibitor of Gli3, a repressor of hedgehog signaling pathway [24]. Nestin tail interacts with Gli3 and prohibits its nuclear translocation, leading a promotion of Gli3 target genes such as Gli1 and $\mathrm{PTCH}$. Because these genes are upstream components of hedgehog signaling pathway promoting cancer proliferation and metastasis [25], we also confirmed their expression level by RNA-seq analysis. As a result, nestin knockout did not alter mRNA expression of both Gli1 and PTCH (Fig. S4B), indicating that reduce of metastasis and invasion ability was not due to the hedgehog signal pathway.

\section{Evaluation of interactions between actin and vimentin}

Cytoskeletal filament proteins interact with each other via linker proteins or intrinsic binding domains. Indeed, the abrogation of plectin, an IF-based cytocrosslinker, decreases the mechanical properties of cells [26]. Nestin copolymerizes with vimentin, which is capable of interacting with actin filaments via its tail domain [27]. Because nestin has a long tail domain $(171 \mathrm{kDa})$ at its C-terminus, it is conceivable that this tail domain could inhibit access of the 7-kDa vimentin tail domain to actin filaments, owing to steric hindrance, resulting from the tail-to-tail configuration formed by the coiled coil structure in the rod domain of IFs. We expressed partial nestin (Nes1-344), which lacks 1520 amino acids of the tail domain and fuses with His tag at the C-terminus, in SNKG8 cells, and measured cellular stiffness by using AFM. The average stiffness of SNKG8 cells expressing the $\mathrm{Nes}_{1-344}(1.86 \pm 1.33 \mathrm{kPa})($ mean $\pm \mathrm{SD} ; n=31)$ was significantly higher than that of the cell expressing the whole nestin fused with His tag at the C-terminus $(1.13 \pm 0.79 \mathrm{kPa} ; n=22)(p=0.008)$. This indicates that $\mathrm{Nes}_{1-344}$ lost an ability to decrease the cellular stiffness by truncation of nestin tail domain. Therefore, the tail domain of nestin is supposed to contribute softening of cancer cell body.

To examine the effect of nestin knockout on intracellular interactions between vimentin and actin filaments, we performed proximity ligation assays (Duolink In Situ PLA). Fluorescent foci derived from proximal actin and vimentin were clearly detected in both the FP10SC2 and SNKG8 (Fig. 3A). Observation of $3 \mathrm{D}$ images obtained via confocal laser-scanning microscopy revealed red spots at both the apical and basal membranes (Movie S1), strongly indicating that vimentin contributes to the maintenance of cellular stiffness by interacting with actin filaments under the cell membrane. The number of fluorescent foci per basal area in SNKG8 cells was approximately two-fold higher than that in FP10SC2 cells (Fig. 3B), showing that interactions between vimentin and actin filaments are inhibited by nestin expression. Thus, the high stiffness of SNKG8 cells is due to increased binding between vimentin and actin filaments, resulting in a reinforcement of the cortical actin under the cell membrane. 
A

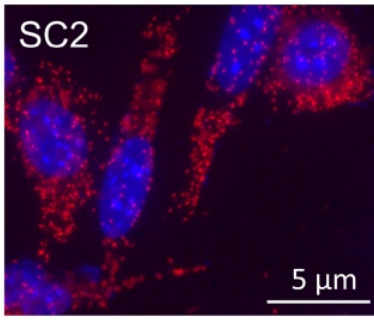

\section{G8}

B

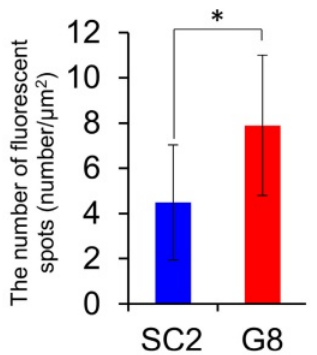

C

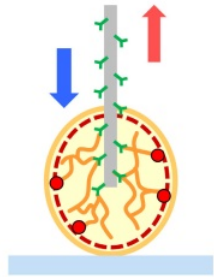

Flexibility: High

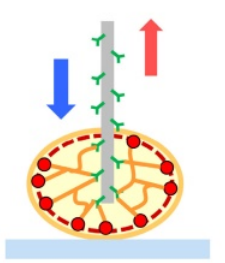

Low

E

D

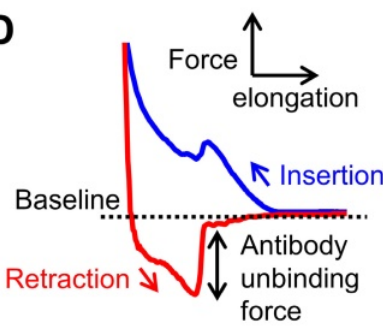

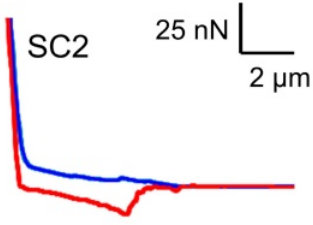

F

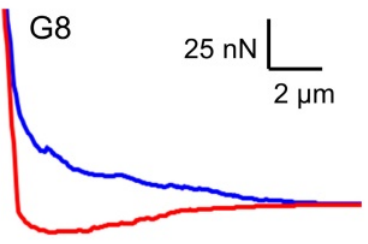

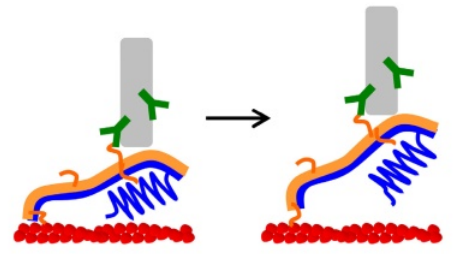
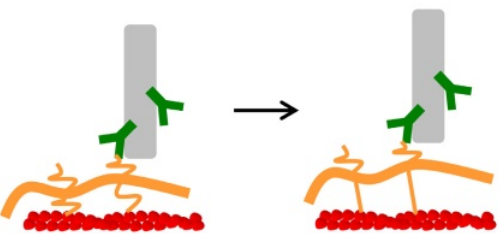

Figure 3. Comparison of the mechanical properties of the cytoskeleton of FP10SC2 and SNKG8 cells. (A) Proximity ligation assay (PLA) analysis for the detection of vimentin-actin interactions. Cells were stained with antibodies specific to actin and vimentin and nuclei were stained with DAPI (blue). Nestin-actin interactions are shown in red. (B) Quantification of the red PLA spots derived from vimentin-actin interactions. The number of spots located on the basal membrane in each group was counted from images of the cells $\left(n=30\right.$ cells) obtained by confocal laser-scanning microscopy and divided by cell area $\left(\mu \mathrm{m}^{2}\right)$, as calculated using Image) software. The results are presented as a means \pm standard deviations; ${ }^{*} \mathrm{p}<0.05$; Student's $t$-test. (C) Tensile test for vimentin by insertion of an antibody-immobilized nanoneedle into individual cells. (D) Force curves obtained by needle insertion and retraction are shown in blue and red, respectively. ( $E$ and $F$ ) Force curve obtained by insertion of an anti-vimentin antibody-immobilized nanoneedle into FPIOSC2 (E) and SNKG8 cells (F). Blue and red lines indicate the approach or retraction of the nanoneedle, respectively. A tensile process of vimentin using nanoneedle is shown in right panel. Nestin, vimentin, and actin filament are illustrated in blue, orange, and red respectively.

\section{Tensile test of vimentin for evaluation of the mechanical properties of the cytoskeleton}

Nestin, by decreasing vimentin-actin binding, could inhibit vimentin accumulation in the cortical actin and alter the flexibility of the cytoskeletal structure. To test this hypothesis, we examined the mobility of IFs in FP10SC2 and SNKG8 cells using AFM and an antibody-modified nanoneedle [28-30]. The nanoneedle fabricated from AFM cantilever is 200 $\mathrm{nm}$ in diameter and $15 \mu \mathrm{m}$ in length. Cell doubling time did not change even after 50 insertions of the nanoneedle [28], indicating that the insertion of 200 $\mathrm{nm}$ nanoneedle into a cell has a little effect on cell viability. By insertion of this nanoneedle into a living cell, cytoskeletal proteins, as antigens, interact with antibodies present on the nanoneedle (Fig. 3C). When the needle is retracted from a cell, cytoskeletal proteins are pulled up. We expect that this tensile test will permit us to evaluate mobility of filaments. If a cell contains flexible cytoskeletal structure because of a low number of binding between cytoskeletal proteins, target cytoskeletal filaments are capable to greatly move during tensile test using nanoneedle (Fig. 3C; Left). In contrast, a mobility of the cytoskeletal proteins using nanoneedle is low in a rigid cytoskeletal structure due to a large number of binding (Fig. 3C; Right). Because nanoneedle is manipulated by AFM, a process of tensile test can be analyzed from a force curve. Figure 3D shows a typical force curve which was obtained during tensile test of cytoskeletal protein using antibody-modified nanoneedle. During the nanoneedle approach process (blue line), the nanoneedle contacts the cell surface and causes an indentation that leads to an increase in the repulsive force exerted on the cantilever. A steep drop in the repulsive force indicates that the nanoneedle has penetrated the cell membrane [31]. When the needle is retracted from the cell (red line), cytoskeletal filaments are pulled and the rupture force of the antibody-antigen binding appear as sharp peaks under baseline (negative force) in a force curve. Local mechanical properties of cytoskeletal proteins can be analyzed by optical tweezers in living cells [32, 
33]. Because a number of antibodies are immobilized on the surface of nanoneedle of over $10 \mu \mathrm{m}$ length, large area of IF meshwork can be captured and pulled simultaneously.

In the current study, we targeted vimentin filaments, that copolymerize with nestin and are expressed in both FP10SC2 and SNKG8 cells. In FP10SC2 cells, the negative force profile which exhibits a process of rupturing multiple interactions between vimentin and antibody increased gradually during the pulling of nanoneedle (Fig. 3E). Because the force curve obtained for nestin using an anti-nestin antibody-immobilized nanoneedle in these cells was similar to that for vimentin (Fig. S5), the nanoneedle functionalized with anti-vimentin antibodies was found to detect IFs composed by nestin and vimentin.

The initial regime of negative force in the force curve obtained in SNKG8 cells is steep and found to be quite different compared to that in FP10SC2 cells (Fig. 3F). This characteristic negative force was repeatedly observed in SNKG8 cells (Fig. S6), suggesting that the difference of the curve profile between FP10SC2 and SNKG8 can be considered an indicator of the mobility of filaments in the cytoskeletal structure. To assess this difference of the force curve, we focused on the intersection of the baseline and the force curve. Because the intersection marks the start of the negative force, the difference of the force curve may be reflected in a differential coefficient at the intersection. Based on this thought, we evaluated the differential coefficient to assess the mobility of the vimentin filaments. The mean differential coefficients obtained from the force curves of FP10SC2 $(n=14)$ and SNKG8 $(n=20)$ cells were $-41.2 \pm 21.1$ and $-57.0 \pm 32.3$, respectively, which were significantly different $(\mathrm{p}<0.05)$. This finding indicates that vimentin filaments in FP10SC2 cells are more flexible than those in SNKG8 cells. Therefore, the differential coefficients at the intersection of these force curves were considered to represent the mobility of the IFs with or without nestin. In FP10SC2 cells, the low binding between actin and vimentin by nestin tail could increase the mobility of the filaments (Fig. 3E). Conversely, cytoskeletal networks consisting of vimentin-actin filaments in SNKG8 cells are difficult to move via retraction of the antibody-functionalized nanoneedle due to the small vimentin tail domain and extensive binding between actin and vimentin (Fig. $3 F$ ). To the best of our knowledge, this is the first report to analyze the mechanical properties of the cytoskeletal architecture of living cells.

\section{Discussion}

In the present study, increase of cellular stiffness by nestin knockout was observed in two types of cells, FP10SC2 and KG-1-C. FP10SC2 has been derived from highly bone marrow metastatic mouse breast cancer cell 4T1E/M3 [18] which is established through in vivo selection of 4T1E cells. KG-1-C which highly expresses nestin is derived from human glioma [34]. Because the origin of these two cell lines such as tissue and organism are quite different, we considered that function of nestin, softening cell body, is not specific to FP10SC2 but general in cancer cell.

We evaluated hematogenous metastasis of nestin knockout cell by animal study. Several studies have reported that nestin expression lowering survival rate of breast cancer patient $[8,10]$. Because effect of nestin knockout, increasing cellular stiffness, may inhibit an invasion of cancer cells that move from primary tumor, knockout or depression of nestin in primary tumor may be a novel therapeutic strategy for inhibition of breast cancer cell metastasis.

In the filamentous structure of IFs, 4 dimers form an octamer, and 4 octamers form a filament composed of 32 IF monomers. Due to the 171-kDa tail domain, nestin could not internally incorporate into filaments and localize to the surface. Previously, in vitro assembly experiments illustrated that the molar ratio of nestin and vimentin in copolymer IFs was approximately 1:4 [1]. This experimental value suggests that approximately 6 monomers of nestin localize to the surface of IFs composed with vimentin and nestin. Because vimentin expression level was not changed by nestin knockout, the effect of nestin on cellular stiffness was caused by the disappearance of surface-exposed nestin tails, which could be included in the vimentin filaments at the same level as that of FP10SC2. These nestin tails on the surface of IFs is considered to cause steric hindrance when the vimentin tail interacts with actin filaments.

The negative force in the force curve obtained by tensile test using antibody-immobilized nanoneedle exhibits a continuous process of rupturing multiple interactions between vimentin and antibody on the surface of nanoneedle. At the intersection of the baseline and the force curve, the multiple interactions are just before ruptured by withdrawal of the nanoneedle. Therefore, differential coefficient at this point is a parameter which is affected by a distribution of target IF in a needle insertion area, the number of antibodies interacting with vimentin, mobility of IF, and rigidity of IF filaments connecting with cytoskeletal network structure. Local density of IF is not uniform, causing a deviation of a differential coefficient which was calculated from a force curve obtained using antibody-modified nanoneedle. However, the differential coefficient correlates with mobility of IF, because the vimentin expression in 
FP10SC2 and SNKG8 cells are same level as shown in the result of western blotting in Fig. 2B. Additionally, the number of antibodies interacting with vimentin can be estimated from a value of work to rupture the interaction between antibody and vimentin, and there is no significant difference between the average value of FP10SC2 and that of SNKG8. These suggest that there are no difference in an average density of IF during the tensile test. Therefore, we suppose the difference of the differential coefficient exhibits a mobility of IF. Although the result of PLA clearly suggests that the mobility of the filament is changed by the reduction of the binding between actin and vimentin, further investigation is needed to reveal an influence of nestin to IF rigidity.

In conclusion, the results of this study indicate that nestin promotes cancer cell invasion and metastasis by enhancing cell softness and flexibility of cytoskeleton. Tensile test and proximity ligation assays revealed that the tail domain of nestin inhibits binding between actin and vimentin, suppressing the accumulation of vimentin at the cortical actin. This could change the mechanical properties of the cytoskeletal structure under the cell membrane and decrease cellular stiffness. Nestin deficiency has been reported not to have a negative effect on mouse development [35]. Since the phenotypes associated with the nestin knockout cell line generated in this work do not contradicted with those observed in human cancer cells, nestin could comprise a viable molecular target for the inhibition of cancer metastasis.

\section{Materials and Methods}

\section{Cell culture}

4T1E [18] and FP10SC2 [17] cells were cultured at $37^{\circ} \mathrm{C}$ in a humidified atmosphere with $5 \% \mathrm{CO}_{2}$ in RPMI 1640 medium (Sigma-Aldrich, St. Louis, MO, USA) supplemented with $2 \mathrm{mM}$ l-glutamine (Wako Pure Chemical Industries, Ltd., Osaka, Japan), $1.5 \mathrm{~g} / \mathrm{L}$ sodium bicarbonate (Invitrogen, Carlsbad, CA, USA), $2.5 \mathrm{~g} / \mathrm{L}$ glucose (Wako Pure Chemical Industries), 10 mM HEPES (Sigma-Aldrich), $1 \mathrm{mM}$ sodium pyruvate (Wako Pure Chemical Industries), and 10\% fetal calf serum (Thermo Fisher Scientific, Inc., Waltham, MA, USA). KG-1-C was cultured in Dulbecco's Modified Eagle's Medium (Sigma-Aldrich) supplemented with $10 \%$ fetal calf serum, $2 \mathrm{mM}$ GlutaMAX (Thermo Fisher Scientific). For cell splitting, cells were treated with PBS containing 0.25\% Trypsin-EDTA (Thermo Fisher Scientific) and then centrifuged to form a pellet. The resulting pellet was dispersed, and the cells were seeded into glass-bottom culture dishes.

\section{Establishment of nestin knockout cells using the CRISPR/Cas9 system}

A CRISPR/Cas9 vector encoding Cas9, GFP, and 20-bp sgRNA sequence designed in the rod domain of mouse nestin amino acid sequence was obtained from Sigma-Aldrich. In this system, complexes of Cas9 protein and sgRNA molecules containing $20 \mathrm{bp}$ sequences homologous to a target gene sequence recognize and bind to that site, and digest the double-stranded DNA. For nestin knockout, we designed an sgRNA that targets the nestin gene located on mouse chromosome 3 to disrupt the rod domain, which plays an important role in forming coiled-coil dimers in the base structure of IFs. FP10SC2 cells were seeded in 24-well plates and transfected with $250 \mathrm{ng}$ of the vector using Lipofectamine 2000 reagent (Thermo Fisher Scientific). Transfected cells were cultivated under G418 selection $(500 \mu \mathrm{g} / \mathrm{ml})$ for $24 \mathrm{~h}$, and single cells were isolated into 96-well plates and maintained in conditioned medium. Knockout of the nestin gene was verified by DNA sequencing analysis, and a lack of nestin expression was confirmed by western blotting and immunostaining analysis. For knockout of human nestin in KG-1-C, a genome editing vector pGedit [36] containing sgRNA sequence (GTCGGCATGCGCCCGCCAGG) was generated as described in the protocol. The KG-1-C cells transfected with the vector was selected by Blasticidin $\mathrm{S}(10 \mu \mathrm{g} / \mathrm{mL})$ for $24 \mathrm{~h}$, and cultured for 7 days in DMEM. Anti-nestin antibody (1:500 dilution, ab18102; Abcam) was used for the immunostaining of human nestin.

\section{Immunostaining}

All of the following procedures were performed at room temperature unless otherwise stated. First, $35-\mathrm{mm} 12 \varphi$ glass base dishes were coated with type 1 collagen and dried. Transfected cells were then seeded into the plates, incubated for $24 \mathrm{~h}$ at $37^{\circ} \mathrm{C}$ in $5 \% \mathrm{CO}_{2}$, washed with PBS three times for 15 min each, and fixed by incubation with $4 \%$ paraformaldehyde for $15 \mathrm{~min}$. After permeabilization with $0.1 \%$ triton X-100 for $2 \mathrm{~min}$, the cells were washed with a blocking solution comprised of $0.4 \%$ Block Ace (DS Pharma Biomedical, Osaka, Japan) in PBS. Cells were then stained with an anti-nestin antibody (1:500 dilution) (Rat-401; BioLegend, San Diego, CA, USA), anti-Clic1 antibody (1:500 dilution) (SC-81873; Santa Cruz Biotechnology, Dallas, TX, USA) or anti-His-tag antibody (1:1000 dilution) (D291-3; MBL, Aichi, Japan) for $1 \mathrm{~h}$, washed three times with $0.4 \%$ Block Ace buffer, and incubated with an Alexa Fluor 488-conjugated goat anti-mouse IgG antibody (1:2000 dilution) (A-11001; Invitrogen) for $1 \mathrm{~h}$. After washing 
three times with $0.4 \%$ Block Ace buffer, cells were incubated with rhodamine-phalloidin stain (1:1000 dilution) (R415; Thermo Fisher Scientific) for $1 \mathrm{~h}$, washed with $0.4 \%$ Block Ace buffer, followed by PBS, and visualized using FV-300 and IX-71 confocal laser-scanning microscopes (Olympus, Tokyo, Japan).

\section{In vivo metastasis}

Seven-week-old female BALB/c mice were purchased from Japan Clea (Tokyo, Japan). For analysis of experimental metastasis, mice were injected with wild type or nestin knockout FP10SC2 cells $\left(1 \times 10^{6}\right.$ cells/mouse $)$ via the tail vein and monitored. They spread through the blood vessels to the lungs and resulted in respiratory failure. Animals were sacrificed when they became moribund. All animal studies were reviewed and approved by the Institutional Animal Care and Use Committee of National Institute of Advanced Industrial Science and Technology (AIST).

\section{Cell invasion assay}

Chemotaxicells (8- $\mu \mathrm{m}$ pore size; Kurabo, Osaka, Japan) coated with Matrigel (BD Bioscience, San Jose, CA, USA) were inserted into 24-well chambers and seeded with $4 \times 10^{4}$ cells in $200 \mu \mathrm{L}$ culture medium containing $0.1 \%$ BSA but no FBS. Meanwhile, the lower chambers were filled with culture medium containing FBS as a chemoattractant. After incubation for $16 \mathrm{~h}$ at $37^{\circ} \mathrm{C}$ in a humidified incubator, cells that migrated to the lower chamber were fixed with $4 \%$ paraformaldehyde for $15 \mathrm{~min}$, stained with $1 \mu \mathrm{M}$ 4,6-diamidino-2-phenylindole (DAPI) (Dojindo Laboratories, Kumamoto, Japan) for $30 \mathrm{~min}$, and enumerated.

\section{Cell motility measurement}

Cell motility was monitored using a cell watcher (COREFRONT Co., Tokyo, Japan). Briefly, cells were seeded on collagen-coated dishes, and the velocity of cell motility was calculated from moving distance of a cell centroid for $30 \mathrm{~min}$. Changes in cell position were recorded every $5 \mathrm{~min}$; at least 20 cells were monitored per strain.

\section{Wound healing assay}

Cells were seeded in ibidi 25 culture-inserts (Minitube Canada, Ingersoll, Ontario) in 35-mm dishes and incubated for $24 \mathrm{~h}$ to generate confluent monolayers. Then, the inserts were carefully removed and $2 \mathrm{~mL}$ of fresh culture medium was added. Dishes were observed using an IX-71 inverted microscope (Olympus) and images were acquired at 0.5, 6, and 12 h. For quantitative analysis, wound surface areas were divided by the length of the observation field to calculate the average distance of the wound $(n=7)$, and the velocity of cell motility was measured by calculating changes in the average wound distance over 6 h. Images were analyzed using Image-Pro Plus software (Media Cybernetics, MD, USA).

\section{Cell elasticity measurement by AFM}

The indenters of cylindrical AFM silicon cantilevers (ATEC-Cont, Nanosensors, Switzerland) were fabricated using a focused ion beam FIB (SMI500; Hitachi High-Tech Science, Tokyo, Japan). The diameter and height of the cylinder were 2.4 and $7.5 \mu \mathrm{m}$, respectively. Spring constants $(\mathrm{k}=0.4 \pm 0.2$ $\mathrm{N} / \mathrm{m}$ ) were determined using the thermal fluctuation method prior to each experiment [37]. The cylindrical tip was operated by a Nanowizard II atomic force microscope (AFM) system equipped with a Cellhesion module, which enables the probe to have a vertical travel distance of up to $100 \mu \mathrm{m}$ (JPK Instruments). Culture dishes were placed on a stage, and force measurements were done within $1 \mathrm{~h}$ after starting the measurement at room temperature. The end-point of force measurement was set at $10 \mathrm{nN}$, and the cylindrical cantilever was driven at a velocity of 10 $\mu \mathrm{m} \cdot \mathrm{s}^{-1}$ during both the approach and retract processes. For measurement of cell body stiffness, the cylindrical cantilever was targeted to the cytosol while avoiding contact to nuclei. For calculation of the Young's moduli $(E)$ from the indentation part of the approach curve, as a parameter of cell stiffness, we used Sneddon's formula [38] for flat-ended cylindrical punch originated from Hertzian contact model:

$$
F=\frac{2 a E}{\left(1-v^{2}\right)} I
$$

where $F, a, E, I$, and $v$ represent force, radius of cylinder $(2.5 \mu \mathrm{m})$, Young's modulus, indentation depth, and Poisson's ratio (0.5 in this study), respectively. To evaluate the stiffness in the surface of the cell, force curves obtained during cell indentation from point of contact with cell to $0.5 \mu \mathrm{m}$ of indentation distance of cantilever were fit by KaleidaGraph (Hulinks, Tokyo, Japan) using this model (Fig. S2B).

For measurement of KG-1-C cells, we used biocompatible anchor for membrane (BAM) conjugated with bovine serum albumin (BSA) [39]. The $27 \varphi$ glass base dish was incubated with $1 \mathrm{mg} / \mathrm{mL}$ BSA in PBS for $3 \mathrm{~h}$ at room temperature. The BSA-coated dish was washed with water 3 times and dried in air. After the addition of $0.5 \mathrm{mM}$ BAM in PBS, the dish was incubated for $30 \mathrm{~min}$ and washed with water 6 times. KG-1-C and nestin knockout cells treated with $0.25 \%$ Trypsin-EDTA were centrifuged to form a pellet and resuspended in PBS. After the centrifugation, cells in DMEM without FBS were 
inoculated on the BAM-BSA dish and incubate for 15 min.

\section{Genetic rescue of nestin in SNKG8}

Mouse nestin expression vector (OmicsLink ${ }^{\mathrm{TM}}$ ORF Expression Clone, EX-Mm04078-M67) purchased from GeneCopoeia (Rockville, MD, USA) was introduced into SNKG8 by use of Lipofectamine 3000 (Thermo Fisher Scientific). The stiffness of SNKG8 cells harboring with nestin expression vector was measured as described above. Nestin expression in the cells after the measurement was confirmed by immunostaining. Fluorescence intensity of the cells was measured by Image-Pro Plus software.

\section{Western blotting}

For preparation of total cellular proteins, cells were washed with ice-cold PBS and collected using a cell scraper. After centrifugation, cell pellets were suspended in RIPA buffer (Santa Cruz Biotechnology) supplemented with protease inhibitor and homogenized by ultrasonication. Lysed cells were centrifuged at $10,000 \times \mathrm{g}$ for $4 \mathrm{~min}$ at $4^{\circ} \mathrm{C}$. The protein concentrations of the lysates were measured using a Pierce BCA protein assay kit (Thermo Fisher Scientific). Approximately $10 \mu \mathrm{g}$ of each sample was then separated by $5-20 \%$ gradient sodium dodecyl sulfate-polyacrylamide gel electrophoresis (SDS-PAGE) and transferred to a polyvinylidene difluoride (PVDF) membrane at $30 \mathrm{~V}$ for $3 \mathrm{~h}$. Membranes were washed with TBS containing $0.1 \%$ Tween 20 (TBS-T) for $5 \mathrm{~min}$ and blocked by incubation with $0.4 \%$ BSA/PBS for $1 \mathrm{~h}$. After rinsing with TBS-T, membranes were treated with the following primary antibodies for $1 \mathrm{~h}$ and rinsed with TBS-T: anti-actin (1:5,000 dilution) (MAB1501R; Millipore, Billerica, MA, USA), anti-a-tubulin (1:3,000) (16-232; Millipore), anti-nestin $\quad(1: 2,000) \quad$ (MAB353; Millipore), anti-vimentin (1:250) (ab28028; Abcam, Cambridge, UK), anti-keratin18 (1:10,000) (ab28028; Abcam), and anti-GAPDH $\quad(1: 15,000) \quad(016-25523 ;$ Wako Pure Chemical Industries). Membranes were then treated with a horseradish peroxidase-conjugated anti-mouse IgG (1:1000) (HAF007; R\&D Systems, Inc., Minneapolis, MN, USA) or anti-rabbit IgG (1:5000 dilution, 074-1506, SeraCare, Milford, MA, USA) secondary antibody for $5 \mathrm{~min}$, and immune complexes were visualized using Clarity Western ECL Substrate (Bio-Rad, Hercules, CA, USA) an enhanced chemiluminescence substrate. Images were obtained by Molecular Dynamics Typhoon device (GE Healthcare, Little Chalfont, UK).

\section{RNA-seq analysis}

Total RNA was extracted using NucleoSpin RNA (TAKARA BIO INC., Shiga, Japan) according to the manufacturer's instructions. Thousand nanograms of RNA was used for library preparation with KAPA Stranded mRNA-seq Kit (KAPA BIOSYSTEMS). The sequenced reads by NextSeq 500 (illumina) were aligned to the mouse genome (GRCm38.p6).

\section{Proximity ligation assay for cytoskeletal proteins}

Proximities between actin and vimentin or nestin filaments were examined by Duolink in situ Proximity ligation assay (PLA) (Sigma-Aldrich). Cells were cultured and fixed with $4 \%$ paraformaldehyde for 15 min. After permeabilization with cold acetone for 1 min, the cells were washed with a blocking solution containing $0.4 \%$ Block Ace (DS Pharma Biomedical) in PBS, incubated with mouse monoclonal anti-actin antibodies (1:400) (AC-40, Sigma-Aldrich, St. Louis, $\mathrm{MO}$, USA) and rabbit monoclonal anti-vimentin (1:500) (EPR3776, Abcam, Cambridge, UK) or rabbit monoclonal anti-nestin antibodies (1:1000) (SAB4200347, Sigma-Aldrich, St. Louis, MO, USA) diluted in $0.4 \%$ Block Ace for $1 \mathrm{~h}$ at room temperature. All other steps were performed according to manufacturer's instructions. To evaluate the number of signals from each PLA reaction, fluorescence spots on the basal membranes of 30 cells were counted.

\section{Evaluation by tensile test of vimentin using an antibody-modified nanoneedle}

Nanoneedles were fabricated from pyramidal silicon AFM cantilevers (ATEC-Cont, Nanosensors) and etched to a cylindrical shape of $200 \mathrm{~nm}$ in diameter and 10-15 $\mu \mathrm{m}$ in length using the FIB [31]. Spring constants $(\mathrm{k}=0.1-0.4 \mathrm{~N} / \mathrm{m})$ were determined as described above. The silicon surface was cleaned with oxygen plasma in a JPA300 plasma asher $(200 \mathrm{~W}$, 5 min) (J-science, Kyoto, Japan) and treated with 1\% $\mathrm{HF}$ for $1 \mathrm{~min}$. After repeating the plasma $(10 \mathrm{~min})$ and $1 \% \mathrm{HF}$ treatment, the nanoneedle was modified by physical adsorption of $50 \mu \mathrm{g} / \mathrm{ml}$ of ZZ-BNC at room temperature for $1 \mathrm{~h}$; ZZ-BNC is bio-nanocapsulebased anchor to which the Fc domain of antibodies can bind [40]. Anti-vimentin (V6630; Sigma-Aldrich) or anti-nestin antibodies (SAB4200347; Sigma-Aldrich) ( $11.75 \mu \mathrm{g} / \mathrm{ml}$ each) were bound to the ZZ-domain of the ZZ-BNC by incubating in PBS overnight at $4^{\circ} \mathrm{C}$. The antibody-immobilized nanoneedle was rinsed prior to use in cell fishing experiments. Force measurements were carried out using the AFM Nanowizard II with CellHesion. For cellular membrane penetration tests, nanoneedles were inserted into cells at an approach velocity of 10 $\mu \mathrm{m} / \mathrm{s}$ with a set point of $200 \mathrm{nN}$, left to dwell within the cells for $60 \mathrm{~s}$, and then evacuated at $10 \mu \mathrm{m} / \mathrm{s}$. Ten 
different sites on each cell were targeted for insertion and three cells were tested for each cell type.

\section{Supplementary Material}

Supplementary figures, table and movie legend.

http://www.ijbs.com/v15p1546s1.pdf

Supplementary movie.

http://www.ijbs.com/v15p1546s2.mov

\section{Acknowledgements}

The authors are grateful to Taro Q. P. Uyeda for useful discussions. This work was supported by the Japan Society for the Promotion of Science (JSPS) KAKENHI Grant Number 26249127, 17H03471, 17K13026, and 19K15373.

\section{Author Contributions}

$\mathrm{CN}$ conceived of the methods and organized the research. MS, YT, MM, MM and AY performed the experiments and data analysis. MI and SK developed ZZ-BNC. TO provided the mouse breast cancer cell line and suggested the research plan used for animal experiments. AY and CN wrote the manuscript. All authors read and approved the final manuscript.

\section{Competing Interests}

The authors have declared that no competing interest exists.

\section{References}

1. Steinert PM, Chou YH, Prahlad V, Parry DAD, Marekov LN, Wu KC, et al. A high molecular weight intermediate filament-associated protein in BHK-21 cells is nestin, a type VI intermediate filament protein - Limited co-assembly in vitro to form heteropolymers with type III vimentin and type IV alpha-internexin. J Biol Chem. 1999; 274: 9881-90.

2. Sahlgren CM, Pallari HM, He T, Chou YH, Goldman RD, Eriksson JE. A nestin scaffold links Cdk5/p35 signaling to oxidant-induced cell death. EMBO J. 2006; 25: 4808-19.

3. Cho HM, Kim JY, Kim H, Sun W. Phosphatase and actin regulator 4 is associated with intermediate filaments in adult neural stem cells and their progenitor astrocytes. Histochem Cell Biol. 2014; 142: 411-9.

4. Mazur AJ, Radaszkiewicz T, Makowiecka A, Malicka-Blaszkiewicz M, Mannherz HG, Nowak D. Gelsolin interacts with LamR, hnRNP U, nestin, Arp3 and beta-tubulin in human melanoma cells as revealed by immunoprecipitation and mass spectrometry. Eur J Cell Biol. 2016; 95: 26-41.

5. Li P, Lee EH, Du F, Gordon RE, Yuelling LW, Liu YQ, et al. Nestin Mediates Hedgehog Pathway Tumorigenesis. Cancer Res. 2016; 76: 5573-83.

6. Herrmann H, Aebi U. Intermediate filaments and their associates: multi-talented structural elements specifying cytoarchitecture and cytodynamics. Curr Opin Cell Biol. 2000; 12: 79-90.

7. Liu CG, Chen B, Zhu J, Zhang RS, Yao F, Jin F, et al. Clinical implications for nestin protein expression in breast cancer. Cancer Sci. 2010; 101: 815-9.

8. Piras F, Ionta MT, Lai S, Perra MT, Atzori F, Minerba L, et al. Nestin expression associates with poor prognosis and triple negative phenotype in locally advanced (T4) breast cancer. Eur J Histochem. 2011; 55: e39.

9. Narita K, Matsuda Y, Seike M, Naito Z, Gemma A, Ishiwata T. Nestin regulates proliferation, migration, invasion and stemness of lung adenocarcinoma. Int J Oncol. 2014; 44: 1118-30.

10. Gao N, Xu H, Liu C, Chen G, Wang X, Li Y, et al. Nestin: predicting specific survival factors for breast cancer. Tumour Biol. 2014; 35: 1751-5.

11. Kleeberger W, Bova GS, Nielsen ME, Herawi M, Chuang AY, Epstein JI, et al. Roles for the stem cell associated intermediate filament Nestin in prostate cancer migration and metastasis. Cancer Res. 2007; 67: 9199-206.
12. Li J, Wang R, Yang L, Wu Q, Wang Q, Nie Z, et al. Knockdown of Nestin inhibits proliferation and migration of colorectal cancer cells. Int J Clin Exp Pathol. 2015; 8: 6377-86.

13. Cross SE, Jin YS, Rao J, Gimzewski JK. Nanomechanical analysis of cells from cancer patients. Nat Nanotechnol. 2007; 2: 780-3.

14. Lekka M. Discrimination between normal and cancerous cells using AFM. Bionanoscience. 2016; 6: 65-80.

15. Liu CY, Lin HH, Tang MJ, Wang YK. Vimentin contributes to epithelial-mesenchymal transition cancer cell mechanics by mediating cytoskeletal organization and focal adhesion maturation. Oncotarget. 2015; 6: 15966-83.

16. Seltmann K, Fritsch AW, Kas JA, Magin TM. Keratins significantly contribute to cell stiffness and impact invasive behavior. Proc Natl Acad Sci U S A. 2013; 110: 18507-12.

17. Okada T, Kurabayashi A, Akimitsu N, Furihata M. Expression of cadherin-17 promotes metastasis in a highly bone marrow metastatic murine breast cancer model. Biomed Res Int. 2017; 2017: 8494286.

18. Takahashi M, Furihata M, Akimitsu N, Watanabe M, Kaul S, Yumoto N, et al. A highly bone marrow metastatic murine breast cancer model established through in vivo selection exhibits enhanced anchorage-independent growth and cell migration mediated by ICAM-1. Clin and Exp Metastasis. 2008; 25: 517-29.

19. Du W, Wang S, Zhou Q, Li X, Chu J, Chang Z, et al. ADAMTS9 is a functional tumor suppressor through inhibiting AKT/mTOR pathway and associated with poor survival in gastric cancer. Oncogene. 2013; 32: 3319-28.

20. Kamekura R, Kolegraff KN, Nava P, Hilgarth RS, Feng M, Parkos CA, et al. Loss of the desmosomal cadherin desmoglein-2 suppresses colon cancer cell proliferation through EGFR signaling. Oncogene. 2014; 33: 4531-6.

21. Kalashnikova EV, Revenko AS, Gemo AT, Andrews NP, Tepper CG, Zou JX, et al. ANCCA/ATAD2 Overexpression Identifies Breast Cancer Patients with Poor Prognosis, Acting to Drive Proliferation and Survival of Triple-Negative Cells through Control of B-Myb and EZH2. Cancer Res. 2010; 70: 9402-12.

22. Oshita H, Nishino R, Takano A, Fujitomo T, Aragaki M, Kato T, et al. RASEF is a Novel Diagnostic Biomarker and a Therapeutic Target for Lung Cancer. Mol Cancer Res. 2013; 11: 937-51.

23. Wang P, Zhang C, Yu P, Tang B, Liu T, Cui H, et al. Regulation of colon cancer cell migration and invasion by CLIC1-mediated RVD. Mol Cell Biochem. 2012; 365: 313-21.

24. Li P, Lee EH, Du F, Gordon RE, Yuelling LW, Liu Y, et al. Nestin Mediates Hedgehog Pathway Tumorigenesis. Cancer Res. 2016; 76: 5573-83.

25. Li X, Deng W, Nail CD, Bailey SK, Kraus MH, Ruppert JM, et al. Snail induction is an early response to Gli1 that determines the effciency of epithelial transformation. Oncogene. 2006; 25: 609-21.

26. Na S, Chowdhury F, Tay B, Ouyang MX, Gregor M, Wang YX, et al. Plectin contributes to mechanical properties of living cells. Am J Physiol Cell Physiol. 2009; 296: C868-C77.

27. Esue O, Carson AA, Tseng Y, Wirtz D. A direct interaction between actin and vimentin filaments mediated by the tail domain of vimentin. J Biol Chem. 2006; 281: 30393-9.

28. Mieda S, Amemiya Y, Kihara T, Okada T, Sato T, Fukazawa K, et al. Mechanical force-based probing of intracellular proteins from living cells using antibody-immobilized nanoneedles. Biosens Bioelectron. 2012; 31: 323-9.

29. Kawamura R, Shimizu K, Matsumoto Y, Yamagishi A, Silberberg YR, Iijima $\mathrm{M}$, et al. High efficiency penetration of antibody-immobilized nanoneedle thorough plasma membrane for in situ detection of cytoskeletal proteins in living cells. J Nanobiotechnology. 2016; 14.

30. Silberberg YR, Mieda S, Amemiya Y, Sato T, Kihara T, Nakamura N, et al. Evaluation of the actin cytoskeleton state using an antibody-functionalized nanoneedle and an AFM. Biosens Bioelectron. 2013; 40: 3-9.

31. Obataya I, Nakamura C, Han S, Nakamura N, Miyake J. Nanoscale operation of a living cell using an atomic force microscope with a nanoneedle. Nano Letters. 2005; 5: 27-30.

32. Guo M, Ehrlicher AJ, Mahammad S, Fabich H, Jensen MH, Moore JR, et al. The role of vimentin intermediate filaments in cortical and cytoplasmic mechanics. Biophys J. 2013; 105: 1562-8.

33. Head DA, Ikebe E, Nakamasu A, Zhang P, Villaruz LG, Kinoshita S, et al. High-frequency affine mechanics and nonaffine relaxation in a model cytoskeleton. Phys Rev E Stat Nonlin Soft Matter Phys. 2014; 89: 042711.

34. Miyake E. Establishment of a Human Oligodendroglial Cell-Line. Acta Neuropathol (Berl). 1979; 46: 51-5.

35. Mohseni P, Sung HK, Murphy AJ, Laliberte CL, Pallari HM, Henkelman $\mathrm{M}$, et al. Nestin is not essential for development of the CNS but required 
for dispersion of acetylcholine receptor clusters at the area of neuromuscular junctions. J Neurosci. 2011; 31: 11547-52.

36. Nagasaki A, Kato Y, Meguro K, Yamagishi A, Nakamura C, Uyeda TQP. A genome editing vector that enables easy selection and identification of knockout cells. Plasmid. 2018; 98: 37-44.

37. Hutter JL, Bechhoefer J. Calibration of atomic-force microscope tips. Rev Sci Instrum. 1993; 64: 1868-73.

38. Sneddon J. Casualties in the casualty department. Practitioner. 1965; 195: 785-9.

39. Kawamura R, Mishima M, Ryu S, Arai Y, Okose M, Silberberg YR, et al. Controlled Cell Adhesion Using a Biocompatible Anchor for Membrane-Conjugated Bovine Serum Albumin/Bovine Serum Albumin Mixed Layer. Langmuir. 2013; 29: 6429-33.

40. Iijima M, Kadoya H, Hatahira S, Hiramatsu S, Jung G, Martin A, et al. Nanocapsules incorporating IgG Fc-binding domain derived from Staphylococcus aureus protein A for displaying IgGs on immunosensor chips. Biomaterials. 2011; 32: 1455-64. 